\title{
Caractères épidémiologiques, cliniques et biologiques des onchocercoses de savane et de forêt en Afrique occidentale
} Revue critique et éléments nouveaux

\author{
par A. PROST, A. ROUGEMONT et M.-S. OMAR \\ Organisation Mondiale de la Santé, B.P. 549, Ouagadougou, Haute-Volta.
}

RÉsumÉ. Une série d'observations nouvelles en Côte-d'Ivoire est l'occasion de passer en revue les facteurs d'environnement, d'hôte, entomologiques, et parasitaires, qui pourraient expliquer l'ampleur des différences épidémiologiques entre le faciès forestier et le faciès de savane de l'onchocercose.

L'ensemble des constatations plaide en faveur de l'existence de deux souches parasitaires, de pathogénicité inégale pour l'homme, inféodées chacune à un groupe de vecteurs différent au sein du complexe $S$. damnosum, et dont les caractéristiques biochimiques ne sont pas identiques. La limite de la forme grave de la maladie est constituée par la limite sud de l'aire d'extension de la paire vectrice $S$. damnosum$S$. sirbanum, au contact de la savane et de la forêt dense. Cette limite géographique et écologique fixe donc à juste titre la terminologie en usage.

Epidemiological, clinical and biological characters of savanna and forest onchocerciasis in west Africa. - Critical review and new data.

SUMmaRy. Recent findings in Ivory Coast provide an opportunity to review possible factors influencing epidemiological differencies between forest and savanna patterns of onchocerciasis.

Environmental factors, host factors, entomological and parasitological factors are taken into consideration. This provides evidence of two strains of parasite, associated with differences in pathogenicity, in biochemical structure, and with different species of the vector complex $\mathrm{S}$. damnosum. The distribution of the severe pattern of the disease covers the area where the savanna vectors $\mathrm{S}$. damnosum s.s. and S. sirbanum prevail, and extends as far as the southern limit of their range. In this respect, the terminology now in use appears to be justified by the geographical extent of the different patterns of the disease.

Accepté le 21 novembre 1979. 
On sait, depuis une brève note de Woodruff en 1957 (21) rapportant son expérience et celle de Murray au Nigéria, que l'onchocercose revêt en Afrique des faciès épidémiologiques différents selon les zones bioclimatiques. Cette notion, très vite simplifiée sous les termes d'onchocercose "de savane " opposée à une onchocercose " de forêt », fut solidement établie au Nigéria et au Cameroun $(1,6,22)$.

Nous-mêmes avons fait des constatations similaires en Afrique de l'Ouest (15) : l'opposition entre la gravité des symptômes dans les régions de savane et leur bénignité en zone forestière $\mathrm{y}$ est telle que la conception unitaire de la maladie peut difficilement être maintenue. En forêt, moins de $4 \%$ des examinés sont porteurs de lésions oculaires graves et les cécités onchocerquiennes sont rarissimes. En savane, à niveau d'infection sensiblement comparable, 10 à $30 \%$ des examinés sont atteints de complications irréversibles; 5 à $12 \%$ de la population est aveugle et l'onchocercose en est responsable dans 6 à 7 cas sur 10. Les complications cutanées et lymphatiques sont elles aussi plus fréquentes en savane.

Cette pluralité des tableaux cliniques doit-elle être attribuée à la coexistence de deux maladies, dues à deux ou plusieurs souches parasitaires de pathogénicité différente, ou bien des facteurs individuels de réceptivité ou d'environnement sont-ils capables d'expliquer une atténuation en forêt de la virulence du parasite ? L'expérience ouest-africaine acquise dans le cadre du Programme de lutte contre l'onchocercose dans le bassin de la Volta éclaire certains aspects du problème et apporte des arguments que nous désirons évoquer au cours d'une brève revue.

Quatre types de facteurs peuvent être mis en cause pour tenter d'expliquer les différences observées: l'environnement, la réceptivité de l'hôte, la transmission par le vecteur, l'adaptation du parasite.

\section{Les facteurs d'environnement.}

Ils sont mis en exergue par la terminologie "savane » et «forêt ", mais nul ne pense que la situation bio-climatique puisse avoir une influence déterminante ni sur l'homme, ni sur le parasite. Seul le vecteur dépend étroitement des conditions écologi. ques favorables ou hostiles à son implantation; l'intensité de l'infection est fonction, dans tous les milieux, de la densité des contacts homme-vecteur. En Afrique de l'Ouest nous n'avons pas mis en évidence de différences sensibles dans l'infection quantitative par Onchocerca volvulus au sein de populations soumises à une forte transmission, qu'elles vivent en savane ou en forêt (15).

L'organisation sociale, l'habitat, les pratiques agricoles modifient localement les caractères épidémiologiques de la maladie. Nous avons montré en savane (14) que la gravité de l'infection et l'intensité des complications, réflétées par le taux de cécité, sont fonction de la densité d'occupation de l'espace : en-dessous d'un seuil de 35 habitants $/ \mathrm{km}^{2}$ utilisé, les communautés en situation hyperendémique sont atteintes par des taux de cécité élevés dépassant souvent $5 \%$ de la population et mettant en question la permanence du groupe. Au-dessus de 50 habitants $/ \mathrm{km}^{2}$ utilisé, la cécité frappe toujours une plus faible proportion des résidents et ne compromet pas le dynamisme du 
groupe. Bien qu'aucune étude similaire n'ait été menée en forêt, rien de semblable ne s'y observe; le taux de complications est aussi faible dans les petits villages que dans les communautés plus importantes.

Les habitudes alimentaires ont été invoquées. Un état de carence nutritionnelle, globale ou sélective, aggraverait l'onchocercose et favoriserait les complications. Les régions d'Afrique sévèrement affectées par la malnutrition protéino-calorique et l'hypovitaminose $\mathrm{A}$ semblent correspondre à celles où sévit l'onchocercose sous sa forme grave. Mais aucun travail n'a encore pu mettre en évidence de façon convaincante le rôle de ces deux facteurs dans la constitution des lésions oculaires graves, ni dans leur évolution vers la cécité $(17,20)$. A l'inverse, rien ne prouve que l'absorption de vitamine A, pas plus qu'une alimentation riche et équilibrée, permette d'éviter la survenue des complications. Enfin, sur le plan épidémiologique, le rôle hypothétique des carences nutritionnelles est incompatible avec l'homogénéité des observations cliniques au sein de vastes régions où l'alimentation de base varie localement et parfois diffère totalement.

\section{Les facteurs d'hôte.}

Les réactions de l'hôte sont sans doute le facteur prépondérant dans certaines situations, soit qu'il y ait évolution foudroyante vers la cécité en quelques années, comme dans l'une de nos observations (délai inférieur à 3 ans chez un enfant de 12 ans), soit qu'une résistance à l'infection se manifeste comme on le constate dans chaque village chez quelques malades. Cette différence de réactivité tient à un ensemble de facteurs individuels mal connus : résistance acquise, susceptibilité différente aux piqûres de simulies, co-existence d'autres infections parasitaires ou bactériennes, autres états morbides associés, dépressions immunitaires générales ou spécifiques, facteurs génétiques, modes de vie, de vêtement ou d'habitat. Aucun n'est à même d'expliquer l'ampleur et la constance des différences observées entre forêt et savane. L'âge de la première infection onchocerquienne, en particulier, n'est pas plus précoce en savane selon nos enquêtes.

Deux faits toutefois méritent attention :

a. Des perturbations importantes de l'immunité cellulaire et humorale ont été observées à plusieurs reprises chez des patients fortement infectés et dans les communautés hyperendémiques de savane. Elles peuvent se manifester vis-à-vis d'antigènes homologues (2), de groupes (5-12) ou hétérologues (4-5).

A cet égard, le phénomène le plus fréquemment relevé concerne le défaut d'intradermoréaction au dérivés protéiniques purifiés (PPD) myco-bactériens. Dans les collectivités hypérendémiques de savane, seuls $25-50 \%$ des sujets réagissent positivement au test, contre $50-85 \%$ dans les villages méso- ou hypo-endémiques $(5,18)$ (fig. 1 et 2 ). De plus, l'hypérendémie de savane est généralement accompagnée d'un excès de valeurs de l'IDR supérieur à $25 \mathrm{~mm}$ (fig. 1) pour lesquelles on peut suspecter une tuberculose en activité (5). 

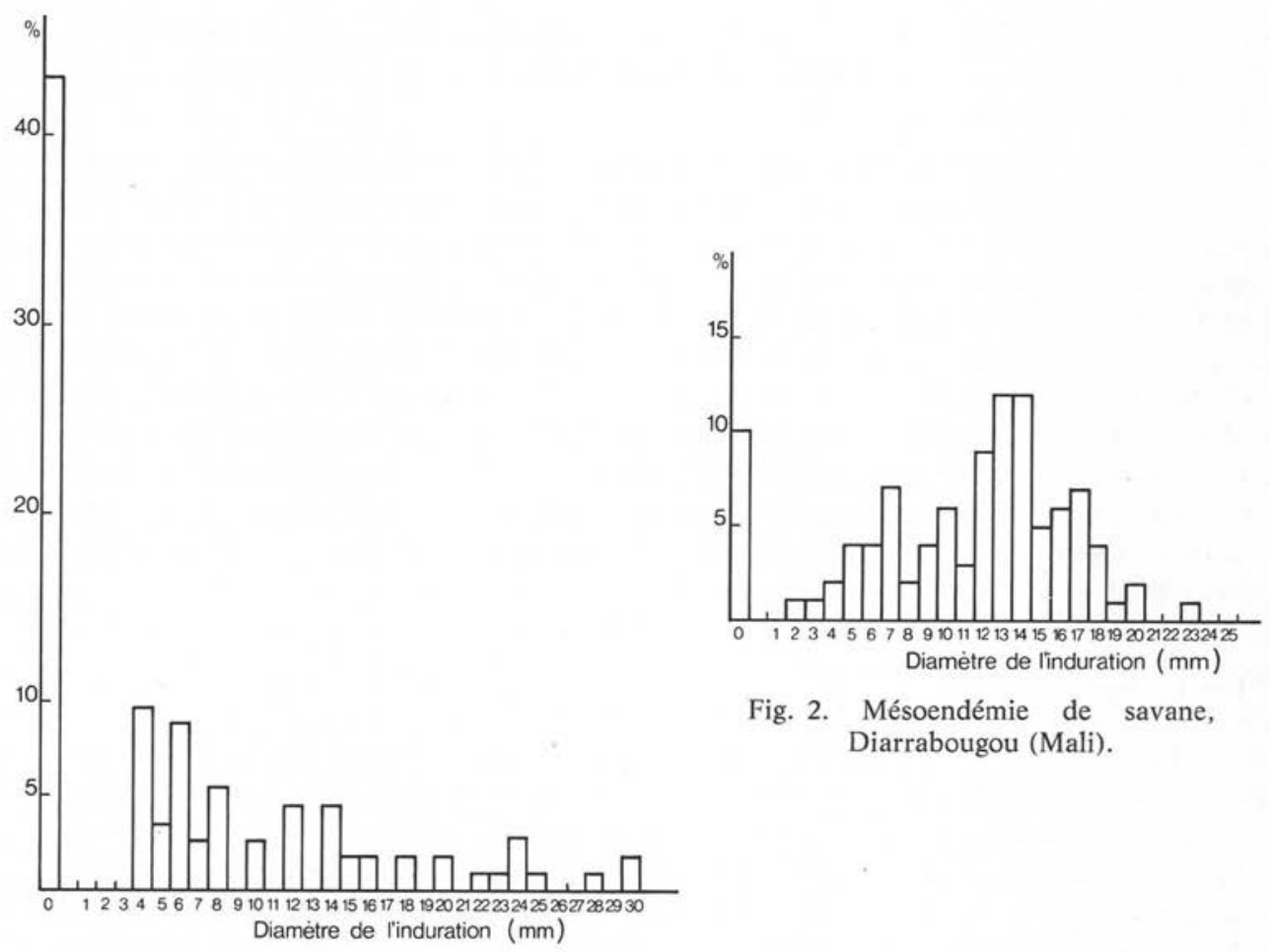

Fig. 2. Mésoendémie de savane, Diarrabougou (Mali).

Fig. 1. Hyperendémie de savane, Koulikoroni (Mali).

Fig. 1 et 2. Distribution de fréquence des intradermo-réactions tuberculiniques dans trois communautés villageoises.

Ces modalités ne se retrouvent pas dans le foyer de forêt étudié en Côte-d'Ivoire où nous avons obtenu $69 \%$ de réponses positives dans le village de Oua (fig. 3) et $74 \%$ dans celui de Sioba, tous deux hyperendémiques.

Ces constatations laissent penser que l'hyperendémie forestière n'entraîne pas de perturbations immunitaires aussi graves que celle de savane.

b. Très peu de malades excrètent des microfilaires dans l'urine en forêt : $2 \%$ à Oua, $3 \%$ à Gbentopleu, 0 à Sioba (foyer ivoirien de Danané), $5 \%$ à Assemanou et $12 \%$ à Blekoum (basse Comoé). Ces chiffres sont très faibles en comparaison de la prévalence de la microfilurie dans les savanes voltaïques et ghanéennes où elle se retrouve chez 20 à $30 \%$ des examinés. Les études en cours tendent à faire de la microfilarurie un symptôme de généralisation de la maladie étroitement corrélé avec la présence de complications oculaires. Il y aurait donc en forêt une meilleure tolérance générale de l'infection onchocerquienne. 


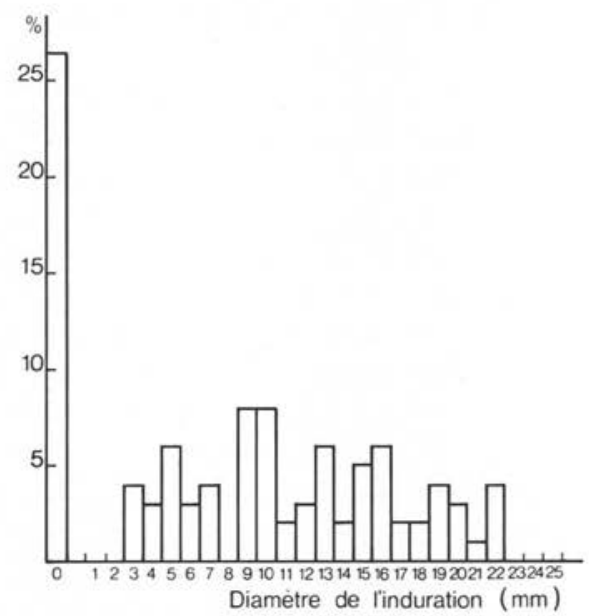

Fig. 3. Hyperendémie de forêt, Oua (Côted'Ivoire).

\section{Les facteurs entomologiques.}

Budden et Le Berre $(6,10)$ avaient suggéré que la dispersion radiaire du vecteur en forêt était responsable d'une transmission moins intense de la maladie et de l'absence de foyers localisés. Le faible taux de complications s'expliquerait alors par une infection moins sévère et une plus faible intensité du parasitisme cutané et oculaire. Sans vouloir discuter l'hypothèse de base, incompatible avec les niveaux de transmission mesurés en Côte-d'Ivoire (1.800 à 2.500 larves infectantes/homme/mois durant une partie de l'année à Oua), nous avons constaté qu'à prévalence et intensité égale du parasitisme, y compris du parasitisme oculaire, le taux des lésions oculaires graves est très inférieur en forêt (15). L'intensité différentielle de la transmission n'est donc pas la cause des variations observées.

Elles ne s'expliquent pas non plus par le pouvoir vecteur des espèces du complexe $S$. damnosum. Au contraire, les espèces forestières ont un rendement parasitaire supérieur à celles de savane (13), le meilleur vecteur étant Simulium yahense, responsable de la transmission dans le foyer de Danané où nous avons diagnostiqué le plus faible taux de lésions cliniques.

Plus importante est l'observation que les villages où sévit une endémie du type forestier sont tous situés au sud de l'aire de répartition saisonnière maxima de Simulium damnosum et Simulium sirbanum (16). L'existence d'une parasitose bien tolérée serait liée à l'absence de ces deux vecteurs de savane et il y aurait association de la gravité de la maladie avec la transmission du parasite par ces espèces. La limite de l'onchocercose de forêt pourrait ainsi être déterminée par les écosystèmes hostiles à l'implantation des simulies de savane. Par contre, nous n'avons constaté aucune différence épi- 
démiologique entre les localités où interviennent l'un ou l'autre des trois vecteurs forestiers (S. yahense, S. soubrense, S. sanctipauli).

Duke avait créé la notion de "Complexe vecteur-parasite » (8) en montrant que des simulies provenant d'une autre région que les onchocerquiens sur lesquels on les infecte sont de plus mauvais vecteurs que dans leur cadre naturel. De même en Côte d'Ivoire, sur des onchocerquiens originaires de Oua (Dt. de Danané), $15 \%$ seulement des simulies s'infectent si elles appartiennent aux espèces $S$. damnosum et $S$. sirbanum naturellement absentes de la région; tandis que plus de $75 \%$ s'infectent si on a recours au vecteur local habituel $S$. yahense ou à l'une des autres espèces de forêt. A l'inverse, le taux d'infection de $S$. yahense reste en-deçà de $15 \%$ si cette espèce est gorgée sur des onchocerquiens venant du Mali, alors que sur les mêmes malades, 45 à $50 \%$ des simulies de savane s'infecteront (16).

Les résultats ne sont pas aussi nets avec tous les vecteurs dans tous les cas. Des situations intermédiaires existent où semble intervenir un facteur limitant qui réduit la fréquence de l'infection mais ne s'oppose pas au cycle intra-vectoriel de tous les parasites. Il est impossible aussi, dans l'état de nos connaissances, de savoir si l'adaptation constatée est réversible. En bref, nous ignorons si ces complexes vecteursparasites témoignent d'une sélectivité de véritables souches parasitaires pour des vecteurs appartenant à des espèces différentes, ou si l'isolement écologique de tels couples a entraîné une simple adaptation, réversible au terme d'une nouvelle confrontation qui verrait s'atténuer progressivement les facteurs limitants.

\section{Les facteurs parasitaires.}

Aucune différence anatomique, histologique, ni antigénique $(3,19)$ entre onchocerques d'origine géographique différente n'avait pu jusqu'ici faire mettre en doute l'unité taxonomique de l'espèce Onchocerca volvulus.

Au cours de nos récentes enquêtes, nous avons étudié l'activité enzymatique des microfilaires de régions différentes par la coloration histochimique des phosphatases acides (11). Les résultats préliminaires étaient encourageants, de même que la spécificité de cette coloration dans le genre Brugia où elle permet de séparer les microfilaires des espèces malayi et pahangi. Nous avons coloré en Côte d'Ivoire 19457 microfilaires prélevées chez 429 onchocerquiens des villages de Sioba, Oua et Nimpleu (foyer du Cavally), Bagro et Nidrou (moyen Sassandra), Assemanou et Blekoum (basse Comoé), appartenant tous au type épidémiologique forestier (15) ; et en Haute-Volta, 11.239 microfilaires prélevées chez 182 malades des villages de Bonga, Niarba et Yakala dans le foyer de savane des Volta Blanche et Rouge. Les résultats (Tableau I) montrent que les microfilaires appartiennent à deux populations différentes. Celles provenant des sept villages forestiers ivoiriens constituent un groupe homogène où dominent les types IV et V (fig. 4) tandis que dans les trois villages de savane voltaïque, les types I et IV se partagent la quasi-totalité des parasites, le type $\mathrm{V}$ étant en pratique absent. Cette répartition n'est nulle part affectée par l'âge ou le sexe des malades. La constance du phénomène au cours d'un grand nombre d'examens itératifs prouve que l'on a à faire à deux populations parasitaires différant au moins par l'un de leurs constituants 
Tableau I. Répartition (en \% du total) des types de coloration histo-chimique des microfilaires de savane et de forêt

\begin{tabular}{|c|c|c|c|c|c|c|}
\hline & $\begin{array}{c}\text { Nombre de } \\
\text { microfilaires } \\
\text { colorées }\end{array}$ & Type I & Type II & Type III & Type IV & Type V \\
\hline $\begin{array}{l}\text { savane }\left(\begin{array}{ll}3 & \text { villages } \\
\text { voltaïques }) & \ldots \ldots \ldots\end{array}\right.\end{array}$ & 11.239 & 17,9 & 2,9 & 3,5 & 75,3 & 0,3 \\
\hline ivoiriens) $\ldots . \ldots \ldots$ & 19.457 & 2,6 & 0,6 & 4,2 & 74,1 & 18,5 \\
\hline
\end{tabular}
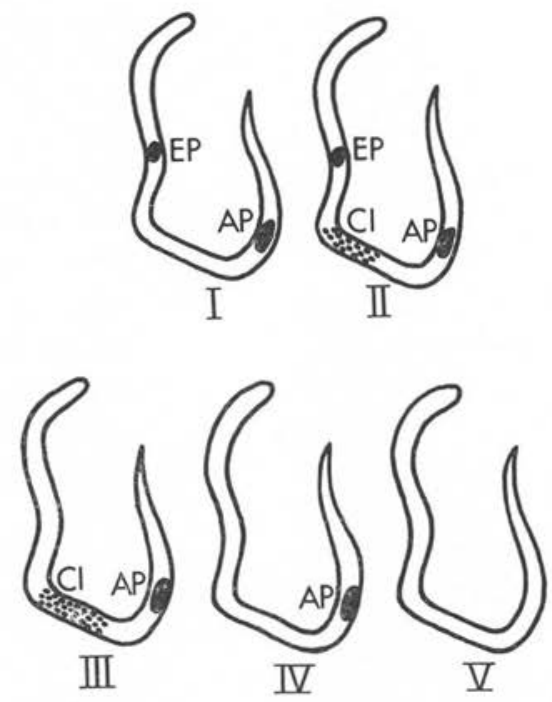

Fig. 4. Cołoration des phosphatases acides chez les microfilaires d'O. volvulus (selon Omar 1978).

AP : Pore anal. EP : Pore excréteur. $\mathrm{CI}=$ Corps interne.

Type I : AP + EP. Type II : AP + EP + CI.

Type III: AP + CI. Type IV: AP seul.

Type V : Coloration négative.

biochimiques. Le caractère ainsi mis en évidence est trop ténu pour constituer déjà un critère de différenciation spécifique ou subspécifique, mais l'association de chacune des deux populations parasitaires avec l'un des deux faciès épidémiologiques reconnus 
plaide en faveur de l'existence de deux souches d'Onchocerca volvulus séparées par leur pathogénicité mais aussi par un ou plusieurs éléments de leur substrat biologique.

Duke $(7,9)$ avait déjà observé que le pouvoir pathogène différentiel était lié au parasite et non à l'hôte ou au «terrain ». L'injection de microfilaires dans le vitrée du lapin avait montré qu'il n'y avait aucune différence entre les lésions postérieures induites par les souches de savane et par celles de forêt; mais l'injection sous-conjonctivale provoquait l'apparition de phénomènes inflammatoires beaucoup plus importants avec les parasites de savane qui avaient une plus grande propension à envahir la cornée.

\section{Conclusion.}

Les enquêtes récentes menées dans le cadre du Programme de lutte contre l'Onchocercose ont montré que cette endémie revêt aussi en Afrique de l'Ouest deux faciès épidémiologiques. Dans l'un, les complications générales sont la règle et se manifestent par une particulière fréquence des lésions oculaires graves, de la cécité, des troubles cutanés, de l'excrétion urinaire de microfilaires, et de la dépression des mécanismes immunitaires. Dans l'autre, à intensité égale du parasitisme, la maladie n'entraîne qu'un très faible nombre de perturbations cliniques et biologiques.

Les résultats présentés plaident en faveur de l'existence en forêt d'une population parasitaire plus faiblement pathogène pour l'homme ; bien adaptée aux vecteurs locaux ; relativement mal transmise par $S$. damnosum et $S$. sirbanum; ayant certains caractères biochimiques propres (activité phosphatasique). A l'inverse, une souche très pathogène en savane paraît inféodée aux vecteurs $S$. damnosum et $S$. sirbanum dont la limite sud d'extension marque aussi la limite de l'onchocercose grave.

Aucun élément ne permet encore de savoir si le degré de pathogénicité est un caractère constitutif du parasite, auquel cas on serait fondé à parler d'un complexe Onchocerca volvulus à l'instar du complexe Simulium damnosum, et à envisager de le démembrer en plusieurs taxons sub-spécifiques ou spécifiques; ou s'il s'agit d'un caractère acquis par atténuation de la souche à la faveur de son entretien par certains vecteurs chez des hôtes plus résistants, et dès lors d'un caractère réversible.

\section{Bibliographie}

1. Anderson J., Fuglsang H., Hamilton P., Marshall T.F. de C.: Studies on onchocerciasis in the United Cameroon republic. II. Comparison of onchocerciasis in rain-forest and Sudan-savanna. Trans. Roy. Soc. Trop. Méd. hyg., 1974, 68, 3, 209-222

2. Bartlett A., Turk J., Ngu J., Mackenzie C. D., Fuglsang H., Anderson J. : Variation in delayed hypersensitivity in onchocerciasis. Trans. Roy. Soc. Trop. Med. Hyg., 1978, 72, 372.

3. Bryceson A.D.M., Van Veen K.S., Oduloju A. J., Duke B. O. L. : Antigenic diversity among Onchocerca volvulus in Nigeria, and immunological differences between onchocerciasis in the Savanna and forest of Cameroon. Clin. Exp. Immunal., 1976, 24, 1, 168-176. 
4. Buck A. A., Anderson R. I., Colston Jr J. A. C., Wallace C. K., Connor D. H., Harman Jr L. E., Donner M. W., Ganley J. P. : Microfilaruria in onchocerciasis. Bull. Wld. Hlth. Org., 1971, 45, 353.

5. Buck A. A., Anderson R. L., Kawata K., Hitchcock Jr C. : Onchocerciasis : some new epidemiological and clinical findings. Amer. J. Trop. Med. Hyg., 1969, 18, 217.

6. Budden F.H.: Comparative study of ocular onchocerciasis in Savanna and rain-forest. Trans. Roy. Soc. trop. méd. hyg., 1963, 57, 1, 64-70.

7. Duke B. O. L., Anderson J. : A comparison of the lesions produced in the cornea of the rabbit eye by microfilariae of the forest and Sudan-savanna strains of Onchocerca volvulus from Cameroon. I. The clinical picture. Tropenmed. Parasit., 1972, 23, 354-368.

8. Duke B. O.L., Lewis J. D., Moore P. J.: Onchocerca Simulium complexes. 1. Transmission of forest and Sudan-savanna strains of Onchocerca volvulus from Cameroon by Simulium damnosum from various west-african bio-climatic zones. Ann. Trop. Méd. Parasitol., 1966, 60, 3, 318-336.

9. Garner A., Duke B. O. L., Anderson J. : A comparison of the lesions produced in the cornea of the rabbit eye by microfilariae of the forest and Sudan-savanna strains of Onchocerca volvulus from Cameroon. II. The pathology. Tropenmed. Parasitol., 1973, 24, 385-396.

10. Le Berre R. : Contribution à l'étude biologique et écologique de Simulium damnosum Theobald 1903 (Diptera, Simuliidae). Mémoires ORSTOM, $n^{\circ}$ 17, 1966, 1 vol., 204 p.

11. Omar M.S. : Histochemical enzyme-staining patterns of Onchocerca volvulus microfilariae and their occurence in different onchocerciasis area. Tropenmed. Parasitol., 1978, 29, 462-472.

12. Ottesen E.A., Weller P.F., Heck L.: Specific cellular immune unresponsiveness in human filariasis. Immunology, 1977, 35, 413.

13. Philippon B.: Etude de la transmission d'Onchocerca volvulus (Leuckart 1893) (Nematoda, Onchocercidae) par Simulium damnosum Theobald 1903 (Diptera, Simuliidae) en Afrique tropicale. Travaux et Doc. de l'ORSTOM, $n^{\circ}$ 63, 1977, 1 vol., 307 p.

14. Prost A., Hervouet J.-P., Thylefors B. : Les niveaux d'endémicité dans l'onchocercose. Bull. Wld. Hlth. Org., 1979, 57, 4, 655-662.

15. Prost A.: Le polymorphisme des onchocercoses humaines ouest-africaines. Ann. Parasitol. Hum. Comp., 1980 (sous presse).

16. Quillevéré D.: Contribution à l'étude des caractéristiques taxonomiques, bioécologiques et vectrices des membres du complexe Simulium damnosum présents en Côte-d'Ivoire. Travaux et documents de l'ORSTOM, 1979, 1 vol., $n^{\circ}$ 109, 304 p.

17. Rodger F.C. : Eye disease in african children with special reference to onchocerciasis and malnutrition. Brit. J. ophtal., 1958, 42, 336-347.

18. Rougemont A., Boisson-Pontal M. E., Pontal P. G., Gridel F., Sangare S.: Tuberculin skin tests and BCG vaccination in hyperendemic area of onchocerciasis. Lancet, febr. 5, 1977, 309.

19. Schacher J.F.: The comparative histo-anatomy of some human strains of Onchocerca volvulus. Proc. 3rd. Int. Congr. Parasitol., München, 1974, 595-596.

20. Wadsworth G. R. : Nutrition in F.C. Rodger «Onchocerciasis in Zaïre », 1 vol. Pergamon Press, Oxford, 1977, 47-68.

21. Woodruff A. W.: Discussion in F.C. Rodger : the pathogenesis of ocular onchocerciasis. Trans. ophtal. Soc., 1957, 77, 286-287.

22. Woodruff A. W., Anderson J., Pettitt L. E., Tukur M., Woodruff A.H. W. : Some aspects of onchocerciasis in Sudan-savanna and rain-forest. J. trop. méd. hyg., 1977, 80, 4, 68-73. 